\title{
ENTRE PASADO Y FUTURO: LA CIENCIA POLÍTICA Y EL PODER LEGISLATIVO EN MÉXICO
}

Alonso Lujambio*

\section{Introducción}

\section{La ciencia política mexicana se ha} negado sistemáticamente a estudiar el Poder Legislativo. Nada pareciera más natural si se piensa que, durante décadas, la composición prácticamente monocolor de las Cámaras del Congreso de la Unión y de los Congresos de los estados de la Federación eliminó del mapa político mexicano el dilema central de la acción colegiada de las asambleas legislativas: la construcción de coaliciones de gobierno. Dicho en otras palabras, y de modo más preciso: el cuadro político postrevolucionario, con mayorías absolutas y calificadas de un sólo partido, el del presidente y el de los gobernadores, en donde además el Ejecutivo funge como líder indiscutible del partido que lo llevó al poder, produjo poderes legislativos inhibidos $\mathrm{y}$, más que incapacitados, desincentivados a echar a andar todo el poder que la Constitución Federal y las constituciones estatales les ofrecieron. Lentamente, el creciente pluralismo de la política mexicana, y su reflejo en la composición partidaria de las asambleas del país, ha ido generando entre los practicantes de

* Profesor de Ciencia Política en el Departamento de Ciencias Sociales del Instituto Tecnológico Autónomo de México (ITAM) y Consejero Electoral ante el Consejo General del Instituto Federal Electoral (IFE). 


\section{ALONSO LUJAMBIO}

la ciencia política un interés más y más profundo sobre la dinámica legislativa. Aún así, la literatura especializada es todavía muy escasa. Este ensayo quiere ser un inventario que discute en tres partes, y a gran escala, lo que sabemos del Congreso mexicano. La primera parte describe a vuelo de pájaro la evolución de los estudios jurídicos sobre el tema. La segunda quiere ser un acercamiento a las historias que se han escrito sobre nuestro objeto de estudio, esto es, a la historiografía del Congreso mexicano. Finalmente, el ensayo intenta hacer un recuento de lo que la ciencia política ha investigado sobre el Poder Legislativo de nuestro país. En la conclusión, se procura apuntalar, con una última reflexión general, la dirección futura de los estudios legislativos en México.

\section{La Ciencia Política, la Ciencia Jurídica y el Congreso Mexicano}

Una visión politológica del Congreso mexicano requiere en primer término de una aproximación jurídica al objeto de estudio. Es imposible entender el proceso político, formal e informal, que pasa por las Cáma-

22 ras, si no se tiene antes que nada una idea clara de lo que las Cámaras deciden según la letra de la Constitución, de su Reglamento Interno y/o de su Ley Orgánica.

En estos campos las aportaciones no son pocas, de modo que cualquier revisión breve de las mismas cometerá injusticias, al omitir este o aquel autor u obra. Sobra decir que para el estudio de las facultades del Congreso mexicano y su evolución histórica, es imprescindible elestudio de diversas obras de Derecho Constitucional, entre las que destaca el clásico de clásicos de Felipe Tena Ramírez, Derecho constitucional mexicano, que viera la luz en el año de 1944 y que para 1992 ya ve en las librerías, bajo el sello de la casa Porrúa, su decimosexta edición. ${ }^{1}$

${ }^{1}$ Ver también otras revisiones como, por ejemplo, Ignacio Burgoa, Derecho constitucional mexicano, 1979, México, Porrúa; Jorge Carpizo, La Constitución mexicana de 1917, 1969, México, Porrúa; y El presidencialismo 
Desde principios de los años setenta se han editado algunas obras colectivas de destacados juristas estudiosos de esta rama específica del Derecho. La primera de ellas fue resultado de una propuesta del entonces diputado Moisés Ochoa Campos, quien puso sus buenos oficios para que se integrara en 1970 una comisión de legisladores y juristas para elaborar el primer tratado de Derecho legislativo mexicano. Ese fue precisamente el título del libro que publicara en 1973 la XLVIII Legislatura (1970-1973), en donde se incluyen ensayos de 16 autores bajo la coordinación, precisamente, del diputado Ochoa Campos. El lector podrá consultar en el libro estudios sobre las facultades del Congreso, sobre las comisiones de ambas Cámaras, sobre la Comisión Permanente, sobre las Mesas Directivas del Senado y de la Cámara de Diputados, así como, muy destacadamente, un detalladísimo estudio del propio Moisés Ochoa Campos sobre las "Fuentes históricas del derecho legislativo mexicano", en donde el autor revisa la evolución histórica de los reglamentos parlamentarios desde el primero de ellos, el del 11 de septiembre de 1813, formulado personalmente por José María Morelos, hasta el Reglamento del 20 de marzo de 1934, todavía vigente, $\mathrm{y}$ sus sucesivas reformas hasta los años sesenta.

Once años después, se publica el segundo volumen colectivo sobre Derecho Legislativo en México. Fue resultado de un coloquio sobre el tema, organizado conjuntamente por la Universidad Nacional Autónoma de México y la LII Legislatura del Senado de la República (1982-1985), en el Colegio de San Ildefonso. El evento tuvo lugar en marzo de 1984. El libro, que llevó por título Política y procesos legislativos, y fue editado por la casa Miguel Angel Porrúa, incluyó 39 ensayos, casi todos muy breves (fueron originalmente ponencias), sobre ocho grandes temas: Situación actual de los Parlamentos, Planeación legislativa, Dinámica legislativa, Práctica legislativa, Diplomacia parlamentaria, El Senado y el federalismo, Integración del Senado y Estudios legislativos.

mexicano, 1978 México, Siglo XXI; y diversos ensayos en Cámara de Diputados de la L Legislatura, Los derechos del pueblo mexicano. México a través de sus Constituciones, 1978, México, Porrúa. 
ALONSO LUJAMBIO

El volumen significaba un avance, porque a pesar del carácter dominante del enfoque jurídico, varios autores ya hacían una diferencia entre los marcos normativos y las prácticas legislativas reales.

Diez años más tarde, se publica el tercer volumen colectivo sobre la temática que aquí nos ocupa. Fue producto esta vez de un Coloquio Internacional organizado por el Instituto de Investigaciones Jurídicas de la Universidad Nacional y la LV Legislatura de la Cámara de Diputados (1991-1994). Ambas instituciones coeditaron el libro El Poder Legislativo en la actualidad, que compiló las 18 ponencias del Coloquio. Se tocaron ahí viejos temas de debate, sobre los que no se hicieron mayores aportaciones, pero también temas que apenas se habían discutido en México, como el de la profesionalización de los legisladores (tema abordado por la estadounidense Susan Rose-Ackerman), los servicios de apoyo al trabajo legislativo (ponencias del mexicano Jesús Rodríguez y Rodríguez y del francés Pierre Avril) y la relación entre sistema de partidos y parlamento (tema abordado por la italiana Carmela De Caro, el español Javier García Roca y los mexicanos Jaime Cárdenas Gracia y Jorge Alcocer).

Otros libros de enfoque jurídico, importantes para la reflexión politológica, son los siguientes. En 1988, nuevamente la casa Porrúa edita el volumen colectivo Técnica legislativa, una obra que discute, con un lenguaje ya muy especializado, el proceso de confección de leyes, más allá de su proceso de creación cameral. El ensayo analiza la estructura que han de seguir las iniciativas de ley, las reglas de redacción de las exposiciones de motivos, las llamadas reglas de supletoriedad y de lingüística legislativa. Un libro importante para todo el que quiere entender de principio a fin el proceso de creación de las leyes.

En segundo lugar, está la obra, editada por el propio autor, Miguel Angel Camposeco, en 1984, titulada Manual de temas legislativos. El libro evoca al de José Romero, publicado por la Cámara de Diputados en 1914, titulado Guía para consultar las prescripciones constitucionales y del Reglamento actual del Poder Legislativo de la Unión, esto es, una especie de diccionario de conceptos relacionados con la labor legislativa, un libro muy útil para los legisladores novicios (es decir, 
para casi todos, si se piensa que en cada Legislatura más del $85 \%$ de los Diputados no tienen experiencia alguna en el campo de la creación de normas) pero también para los estudiosos del proceso legislativo, en la medida en que condensa en un solo volumen información que se encuentra dispersa en una gran diversidad de fuentes.

Otro libro dedicado a los aspectos jurídicos generales de la actividad parlamentaria, es el de Francisco Berlín Valenzuela, Derecho parlamentario, editado por el Fondo de Cultura Económica en 1993. El libro incluye información muy útil de derecho comparado.

Por último, cabría mencionar algunos estudios jurídicos, y no soy exhaustivo aquí para no agobiar al lector, que sin duda resultarán útiles al politólogo que quiera especializarse en temas de política legislativa. En primer lugar, sobre las comisiones legislativas existen dos estudios imprescindibles con enfoque jurídico. Me refiero a los de José Antonio González Fernández, "Las Comisiones del H. Congreso de la Unión" (en Obra Jurídica Mexicana, PGR, 1988), que combina los enfoques jurídico e histórico, y el de Luis Raigosa Sotelo, "Las Comisiones parlamentarias en el Congreso mexicano" (Revista Mexicana de Estudios Parlamentarios, I, 3, 1991), con importantes reflexiones de derecho comparado.

Sobre normatividad interna, agregaría a lo ya expuesto el ensayo de José Barragán Barragán, "Algunas consideraciones de carácter histórico sobre el régimen interno del Congreso de la Unión" (Revista Mexicana de Estudios Parlamentarios, I, 2, 1991). Sobre la reforma de 1994 a la Ley Orgánica del Congreso (creada en 1979 y apenas reformada en 1981), véase el excelente, aunque breve, estudio de uno de sus creadores, el entonces diputado Miguel González Avelar, "La nueva Ley Orgánica del Congreso" (Quórum, IV, 31,1994$)$. ${ }^{2}$ Sobre la evolución postrevolucionaria, de orden estrictamente constitucional, del Congreso mexicano, existen dos ensayos, el de José de J. Orozco Enríquez, "El Poder Legislativo en el Constituyente de Querétaro y

${ }^{2}$ Véase el Diario Oficial del 25 de mayo de 1979, del 28 de diciembre de 1981 y del 20 de julio de 1994. 


\section{ALONSO LUJAMBIO}

su evolución posterior" (Estudios jurídicos en torno a la Constitución mexicana de 1917 en su septuagésimo quinto aniversario, UNAM, s/f) y el de Diego Valadés, "Las transformaciones del Poder Legislativo mexicano" (Obra jurídica mexicana, PGR, 1988). Una propuesta muy reciente de reformas a los mecanismos de control del Legislativo sobre el Ejecutivo se encuentra en el capítulo cuarto del libro de Jaime Cárdenas Gracia, Una Constitución para la Democracia. Propuestas para un nuevo orden constitucional (México: UNAM, 1996). La obra con enfoque jurídico más reciente, de Susana Thalía Pedroza, El Congreso de la Unión: integración y funciones (México: UNAM-IIJ, 1997) tiene un carácter esencialmente descriptivo pero valioso, ya que incluye consideraciones sobre las últimas reformas a la Ley Orgánica del Congreso en 1994.

Como corolario, aclaro al lector que las referencias bibliográficas que encuentre citadas en estas obras pueden ofrecer, en el conjunto, una visión prácticamente exhaustiva del análisis jurídico de nuestro Poder Legislativo.

\section{Ciencia Política, Historia y Poder Legislativo en México}

El politólogo que quiera involucrarse de lleno en la tarea de investigar el Poder Legislativo mexicano debe revisar la literatura histórica sobre el tema. No podemos dejar de subrayar que los historiadores mexicanos no se han sentido particularmente atraídos por el Congreso. Como se verá, no existe un gran volumen de literatura, prácticamente sobre ninguna etapa de nuestra historia, salvo quizá, como veremos aquí, la muy breve de la presidencia de Francisco I. Madero, en la que el Congreso jugó un papel determinante.

Dos investigaciones históricas de gran envergadura han estudiado, casi siempre de paso, el Poder Legislativo. Me refiero a la magna obra coordinada por Daniel Cosío Villegas, Historia moderna de México (México: Hermes, varios tomos publicados entre 1955 y 1965), que estudia la República Restaurada y el Porfiriato, en donde encontramos 
CIENCIA POLÍTICA Y PODER

referencias constantes a la vida parlamentaria de la época. Con base en aquella investigación, Cosío Villegas publicó a finales de los sesenta un elegante ensayo, "El Congreso vigilado", en donde se analizan muy puntualmente tres Legislaturas del Congreso, la VIII (1876-1878), la IX (1878-1880) y la X (1880-1882), para sostener la hipótesis del inicio del ocaso parlamentario después de la Revolución de Tuxtepec en 1876 (Revista Mexicana de Sociología, XXXI, 1, 1969). ${ }^{3}$ Cabe mencionarse también la obra colectiva Historia de la Revolución mexicana 1911-1960, ambiciosa empresa de El Colegio de México coordinada en su origen por Daniel Cosío Villegas, que incluye dos decenas de tomos publicados a finales de los años setenta. Distintos autores analizan en diversos volúmenes diferentes momentos del Congreso en la Revolución y en la era postrevolucionaria, particularmente Luis Medina Peña en los volúmenes 18 y 20 dedicados al período 1940-1952.

Pero procedamos en orden. Del período que va de la primera República Federal (1824-1834) a la Revolución de Ayutla (1855) sabemos poco. Obras generales como los clásicos de Michael P. Costeloe, La Primera República federal en México, 1824-1834 (México: Fondo de Cultura Económica, 1975) y de Moisés González Navarro, Anatomía del poder en México, 1848-1953 (México: El Colegio de México, 1977) nos ofrecen pistas pero nunca una visión integral o por lo menos una hipótesis sobre el papel que el Congreso jugó en aquellos años. ${ }^{4}$ Casi quince años después de que su libro viera la luz, el propio Costeloe publicó un breve ensayo sobre el conflicto entre los poderes Ejecutivo y Legislativo en los primeros veinte años de la vida independiente de

${ }^{3}$ No olvidemos que el consecutivo de nuestras Legislaturas no comienza con la Constitución de 1917 sino con la de 1857.

${ }^{4}$ Una fuente de consulta obligada, sin embargo, es la monumental compilación, a la que el autor dedicó 35 años, de Juan A. Mateos, Historia Parlamentaria del Congreso Mexicano, 1822-1856 (México: varios editores entre 1877 y 1912). Por otro lado, la extraordinaria obra de Lucas Alamán aborda de modo disperso reflexiones sobre el Congreso Mexicano en la primera mitad del siglo XIX. 


\section{ALONSO LUJAMBIO}

México, en donde plantea la hipótesis de que el cleavage civiles vs. militares se traducía institucionalmente en un conflicto entre poderes: si bien los generales dominaron la ruta de acceso al Poder Ejecutivo, por otro lado fueron especialmente incapaces de controlar al Congreso, que estuvo siempre compuesto por mayorías de civiles electos ("Generals vs. Politicians: Santa Anna and the 1842 Congressional Elections in Mexico", Bulletin of Latin American Research, VIII, 2, 1989). Por otro lado, y si bien esta obra concentra su atención en el análisis de los poderes extraordinarios del presidente en el período en comento, The Liberal Cloak. Emergency Powers in XIXth Century Mexico, de José Antonio Aguilar, constituye una importante aportación al análisis de esta esfera de la relación Ejecutivo-Legislativo en el siglo XIX (Chicago University, tesis de Doctorado en Ciencia Política, 1996).

Sin embargo, dos extraordinarias obras dedican su atención al Congreso en dos momentos específicos de nuestro siglo XIX. Una es la de Reynaldo Sordo Cedeño, El Congreso en la Primera República Centralista (México: El Colegio de México-Instituto Tecnológico Autónomo de México, 1993), que analiza el período 1833-1841, es decir, que corre del ocaso del primer gobierno liberal, el de Valentín Gómez

28 Farías (1833-1834), durante el cual el Congreso está dominado por una facción, ya, de liberales puros, al término del tercero -y últimogobierno de Anastasio Bustamante (1839-1841), cuando el Congreso empieza a ser testigo de nuevas alianzas entre conservadores y liberales moderados. En segundo lugar, está la obra de Cecilia Noriega Elío, El Constituyente de 1842 (México: UNAM, 1986), que estudia el breve pero interesantísimo período entre el levantamiento de Paredes y Arrillaga en 1841 y la promulgación de las Bases Orgánicas de 1843). El Congreso Constituyente de 1842, que como se sabe nunca logró sancionar su obra, destaca por la elevada calidad de sus debates, en donde distintas tendencias políticas en conflicto intentan repensar una conciliación a través de un nuevo arreglo constitucional. La autora analiza, entre otras cosas, datos comparativos (edad, ocupación, procedencia, etc.) del Constituyente de 1842 y del de 1843, y logra expli- 
car el nuevo giro conservador que la política mexicana diera una vez fracasada la tarea del Constituyente de 1842.

Pero el ensayo más ilustrativo sobre el período, exquisito a mi modo de ver, aunque extremadamente breve, es, también, de Reynaldo Sordo Cedeño, "El Congreso y la formación del Estado-Nación en México, 1821-1855" (en Josefina Vázquez, coord., La fundación del Estado mexicano, México, Nueva Imagen, 1994). Sordo describe el período como "la época de oro del Poder Legislativo Mexicano", en la que dicho poder es mucho más estable que el Ejecutivo: hubieron en el período veintidós Congresos, pero el Ejecutivo cambió cuarenta y cinco veces de manos (diez veces fue Santa Anna). Por otro lado, entre 1824 y 1855 , es decir, en el espacio de treinta y un años, solamente durante cinco años y cinco meses no hubo Congreso en México (de los cuales dos años y cuatro meses corresponden a la última dictadura de Santa Anna, de abril de 1853 a agosto de 1855), a diferencia de otros países latinoamericanos, como Argentina bajo Rosas, en donde el caudillismo como dictadura eliminó del mapa político al Poder Legislativo durante largos períodos. Sordo analiza en su ensayo, entre otras cosas, los conflictos entre el Congreso y los presidentes Santa Anna, Bustamante, Paredes y Arrillaga, Herrera y Arista (quien renuncia a la presidencia el 5 de enero de 1853, frente a un Congreso ya francamente obstruccionista).

Sobre el Constituyente de 1857 destaca, para un estudio de los debates, las fuentes primarias de Francisco Zarco, Historia y Crónicas del Congreso Constituyente, 1856-1857 (México: El Colegio de México, 1956 y 1957). El clásico de clásicos, crítico de la obra de aquel Congreso, es el de Emilio Rabasa, La Constitución y la dictadura (México, 1912, reimpreso en 1957 por Porrúa). Una brillante defensa del Congreso Constituyente de 1857 se encuentra en Daniel Cosío Villegas, La Constitución de 1857 y sus críticos (México: Hermes, 1957). Un excelente análisis estadístico de la composición social de dicho Congreso y de la conducta legislativa de sus miembros, por cierto sorprendentemente ignorado por los estudiosos mexicanos del tema, es el del norteamericano Richard N. Sinkin, "The Mexican 
ALONSO LUJAMBIO

Constitutional Congress, 1856-1857: A Statistical Analysis" (The Hispanic American Historical Review, LIII, 1, 1973). Pero a mi modo de ver, el mejor análisis del arreglo constitucional de 1857 y de sus consecuencias en términos de la relación, conflictiva casi siempre, entre el Ejecutivo y el Legislativo bajo las presidencias (en períodos de paz) de Juárez y de Lerdo, es el de quien fuera precisamente biógrafo de Sebastián Lerdo de Tejada, Frank A. Knapp, Parliamentary Government and the Mexican Constitution of 1857: A Forgotten Phase of Mexican Political History" (The Hispanic American Historical Review, XXXIII, 1, 1953). Ahí el autor analiza cómo el Congreso Constituyente de 1857 decide retirarle al Ejecutivo la capacidad de iniciativa y le deja un veto suspensivo prácticamente inútil, en buena medida como reacción a la última dictadura de Santa Anna. Lo natural entonces hubiera sido que el presidente, o por lo menos su gabinete, dependiera de la confianza del Congreso (con la Cámara de Diputados como cámara única). Pero la Constitución de 1857 ofreció al presidente plena libertad para nombrar y remover a los miembros de su gabinete, dando como resultado una permanente fricción entre poderes en la definición de la integración del gabinete presidencial. Finalmente, sobre el Congreso entre el Constituyente de 1857 y el arranque del Porfiriato está la obra, aún inconclusa, de Fernando Dworak, en donde se analizan la inestabilidad del gabinete presidencial a partir de la compleja relación Ejecutivo-Legislativo, las tasas de reelección durante la República Restaurada y la evolución del sistema de comisiones legislativas, entre otras variables (El Congreso de la Unión, 1857-1880, ITAM, Departamento de Ciencias Sociales, tesis de Licenciatura en preparación).

Del Congreso durante el Porfiriato sabemos poco. Más allá de los datos que nos ofrece Cosío Villegas en las obras ya citadas, que por cierto dan sustento a la hipótesis de un Congreso completamente subordinado al dictador, se encuentran las obras de François-Xavier Guerra, México. Del Antiguo Régimen a la Revolución (México: Fondo de Cultura Económica, 1988), que toca el tema de paso, y sobre todo el de Marcello Carmagnani, Estado y Mercado. La economía política del liberalismo mexicano, 1850-1911, en donde el autor analiza la política 
presupuestal del Porfiriato y concluye, entre otras muchas cosas, que no fue sino a partir de la última década del siglo XIX, y no antes, cuando el debate parlamentario perdió centralidad en las definiciones presupuestales del régimen. Sobre la reelección en el Porfiriato, mítica por lo demás, que llevara a Cosío Villegas a describir al Congreso de la época como "un museo de Historia Natural", se encuentra en proceso el estudio de Sophie Sosa Palazuelos, La reelección en el Congreso durante el Porfiriato (ITAM, Departamento de Ciencias Sociales, tesis de Licenciatura en preparación). Una de las conclusiones preliminares a las que ya arriba Sosa es que la reelección en el Porfiriato experimentó distintas tasas en distintos momentos, llegando a su clímax en el período 1892-1998, precisamente cuando, según el estudio de Carmagnani, el Congreso comienza su definitivo ocaso.

Pero es sin duda la XXVI Legislatura, la de la presidencia de Madero, la que ha despertado mayor interés entre los historiadores. Fue por lo demás un momento de gran entusiasmo sobre los nuevos alcances de la actividad parlamentaria en México, actitud que condujo a debates congresionales como aquel que planteara ardientemente si miembros del gabinete de Madero debían o no comparecer ante la representación nacional, y por lo tanto volverse o no sujetos de una censura, no importa si formal o no, por parte del Congreso. El debate no se limitó a los miembros de la XXVI Legislatura. También invadió el mundo de la discusión intelectual, tal como lo constatan las obras de, en primer lugar, Antonio Enríquez, Dictadura presidencial o parlamentarismo democrático (México: Imprenta de A. Enríquez, 1913), quien argumenta en favor del parlamentarismo, "principalmente porque aleja toda posibilidad de mantener o crear caudillajes y dictaduras como las que hemos tenido". Y, en segundo lugar, la de Manuel Cruz, que critica, según él por ignorantes, a los defensores del parlamentarismo, en su obra El Gobierno de gabinete y la evolución del parlamentarismo en Inglaterra (México: Imprenta Franco-Mexicana, 1918), en donde se argumenta en contra de "pruritos innovadores" que terminan en "utopías irrealizables". De hecho, Cruz, quien escribe el prólogo de su obra en abril de 1918, no sólo responde a Enríquez sino a los enemi- 
ALONSO LUJAMBIO

gos de Carranza, que presentan en el Congreso, apenas vigente la todavía no mítica Constitución de 1917, esto es el 15 de diciembre de 1917, una sofisticada iniciativa de reforma constitucional para llevar a México del presidencialismo al parlamentarismo o, mejor dicho, a una especie de semi-presidencialismo parlamentario al estilo de lo que poco después se vería en la República de Weimar o, ya en los cincuenta, en la V República Francesa. ${ }^{5}$

Pero volvamos a nuestra materia. Existen tres obras sobre la XVII Legislatura. La de Jorge Sayeg Helu, Significación histórico-politica de la Cámara de Diputados de la XXVI Legislatura Federal (México: Instituto Nacional de Estudios Históricos de la Revolución Mexicana, 1979), de carácter descriptivo, retorna esencialmente lo que ya Diego Arenas Guzmán nos había ofrecido en los cinco tomos de su Historia de la Cámara de Diputados de la XXVI Legislatura Federal (México: Instituto Nacional de Estudios Históricos de la Revolución Mexicana, 1961 a 1966). Las otras son más analíticas, la de Josefina MacGregor, La XXVI Legislatura. Un episodio de la historia legislativa de México (México: Cámara de Diputados, 1983) y la de Pablo Picatto, Congreso y Revolución (México: Instituto Nacional de Estudios Históricos de la Revolución Mexicana, 1991). Sobre las elecciones que dan origen a la XXVI Legislatura, está el estupendo ensayo de François-Xavier Guerra, "Las elecciones legislativas de la Revolución mexicana: 1912" (Revista Mexicana de Sociología, LII, 2, 1990) que analiza con gran detalle las dificultades de un orden democrático en el México que Francisco I. Madero pretendía transformar.

${ }^{5}$ Se presentó otra iniciativa en el mismo sentido pero mucho más simple, el 25 de noviembre de 1921. Alonso Lujambio y Jeffrey Weldon, "La Constitución de 1917 y el Parlamentarismo", ITAM, ensayo en preparación: Huelga decir que, en abril de 1916, la Soberana Convención Revolucionaria de Aguascalientes también se pronunció en contra del régimen presidencial y a favor del parlamentarismo. 
CIENCIA POLÍTICA Y PODER

\section{La Ciencia Política del Congreso Mexicano}

Una gran diversidad de obras, tanto de politólogos mexicanos como de extranjeros, han tocado muy de paso el tema del Poder Legislativo en la era postrevolucionaria. En una de las obras clave de la historia de la ciencia política en México, La Democracia en México, de Pablo González Casanova (México: Era, 1965), se ofrecen datos empíricos sobre producción legislativa, de origen tanto presidencial como congresional, para arribar a la conclusión de que "el sistema de equilibrio de poderes no funciona", de que el Legislativo "simplemente sanciona los actos del Ejecutivo". Frente a estos hechos, los estudios legislativos no parecían inflamar el entusiasmo de nadie. Otros temas atrajeron la atención de los investigadores.

El mexicano-norteamericano Rudolph O. de la Garza rompe con el desinterés de la disciplina en la materia, al escribir en 1972 una obra dedicada íntegramente al Congreso mexicano, The Mexican Chamber of Deputies and the Mexican Political System (Universidad de Arizona, tesis de Doctorado). De la Garza observa al Congreso de México bajo la óptica del estructural-funcionalismo, teoría dominante en la ciencia política norteamericana en aquellos años. La contribución más importante de dicha obra tiene que ver con la "función" del Congreso como ámbito de reclutamiento de las élites políticas postrevolucionarias. La separata de la obra general que discute este tema puede consultarse en "La función reclutadora de la Cámara de Diputados" (Revista Mexicana de Ciencia Política, XXI, 1975).

Después de la obra de De la Garza, tendríamos que esperar prácticamente dos décadas para conocer nuevas contribuciones extensas, con ambición analítica, de la ciencia política al conocimiento de nuestro Poder Legislativo. Una obra ambiciosa, completamente dedicada a uno de los temas más complejos y peculiares del Congreso en México, es la de Benito Nacif Hernández, The Mexican Chamber of Deputies. The Political Significance of Non-Consecutive Re-election (University of Oxford, tesis de Doctorado en Ciencia Política, 1995). El estudio 
ALONSO LUJAMBIO

de Nacif analiza las carreras políticas de los parlamentarios mexicanos del partido en el poder para detectar el modo en que los incentivos institucionales están colocados (y particularmente la prohibición de la reelección inmediata) para mantener sistemáticamente débil el Poder Legislativo.

Sobre el tema particular del origen, evolución y consecuencias de la devastadora regla de la no-reelección se han escrito extraordinarios ensayos en los últimos años, que complementan, enriquecen o corrigen, algunas de las ideas de Nacif. Emma Campos analiza empírica y exhaustivamente, para el período 1934-1997, el modo tan precario en que las dos cámaras del Congreso mexicano reclutan ex-legisladores federales y locales en Un Congreso sin congresistas. La no-reelección consecutiva en el Poder Legislativo mexicano, 1934-1997 (ITAM, Departamento de Ciencias Sociales, tesis de Licenciatura en Ciencia Política, 1996). Igualmente, Maite Careaga estudia el intento frustrado de modificar la regla de la no-reelección a mediados de los sesenta, cuando en 1964 la Cámara de Diputados aprobó la reforma al 59 constitucional, pero el Senado la rechazó en 1965, en Reformas institucionales que fracasan. El caso de la reforma reeleccionista en el Congreso

34 mexicano, 1964-1965 (ITAM, Departamento de Ciencias Sociales, tesis de Licenciatura en Ciencia Política, 1996). Un excelente análisis de los orígenes históricos de la prohibición se encuentra en Jeffrey Weldon, "Congress, Political Machines, and the Maximato: The No-Reelection Reforms of 1933" (ponencia presentada ante la Latin American Studies Association en Atlanta, Georgia, marzo de 1994).

Sin embargo, la fórmula electoral, la que define la integración de las Cámaras, fue objeto de investigación desde mediados de los ochenta, y aún antes, aunque fuera muy en la coyuntura, ${ }^{6}$ desde que la reforma

${ }^{6}$ Ver Miguel de la Madrid Hurtado, "Reformas a la Constitución Federal en materia de representación" en Revista de la Facultad de Derecho de la UNAM, XIII, abril-junio de 1963, Mario Moya Palencia, La reforma electoral (México: Plataforma, 1964) y Vicente Fuentes Díaz, Origen y evolución del Sistema Electoral (México: edición del autor, 1967). 
CIENCIA POLÍTICA Y PODER

electoral del 28 de diciembre de 1963 posibilitara la representación de los partidos minoritarios en la Cámara de Diputados con lo que entonces se llamara "los diputados de partido". Caben mencionarse aquí las obras de Juan Molinar Horcasitas, "The Mexican Electoral System: Continuity by Change" (en Paul Drake y Eduardo Silva, eds., Elections and Democratization in Latin America, San Diego, UCSD, 1986); Alonso Lujambio, La proporcionalidad política del Sisterna Electoral Mexicano, 1964-1985 (ITAM, Departamento de Ciencias Sociales, tesis de Licenciatura en Ciencias Sociales, 1987) y, más recientemente, Leonardo Valdés Zurita, Las consecuencias políticas de las reformas electorales en México, 1978-1991 (E1 Colegio de México, tesis de Doctorado en Ciencias Sociales, 1993).

Sobre el sistema de comisiones, la literatura sólo cuenta con un puñado de obras. Está todavía en proceso una ambiciosa investigación de Cecilia Martínez Gallardo, El sistema de comisiones en la historia del Congreso mexicano (TTAM, Departamento de Ciencias Sociales, tesis de Licenciatura en preparación), de modo que aún no tenemos una visión panorámica. En mi ensayo "La Cámara de Diputados en México. Arreglos Institucionales y Proceso Político" (en Alonso Lujambio, Federalismo y Congreso en el cambio político de México, UNAM-IIJ, 1995) he analizado en perspectiva comparada el sistema mexicano de comisiones legislativas, con el fin de detectar el origen de su debilidad, y he proporcionado evidencia empírica de su evolución desde finales de los años ochenta: el tamaño de las comisiones, su composición partidaria, la titularidad partidaria de sus presidencias y secretarías, etc.

Por otro lado, existen hasta donde llega mi conocimiento solamente dos estudios sobre comisiones específicas. Uno es el de Jorge Padilla Jiménez de la Cuesta, El Congreso: la inconexión electoral. La Comisión de información, gestoría y quejas de la Cámara de Diputados en México (ITAM, Departamento de Ciencias Sociales, tesis de Licenciatura en Ciencia Política, 1995) en donde el autor analiza la notoria e impresionante debilidad de la relación entre los diputados y sus representados en distritos uninominales y el modo en que esa precaria rela- 


\section{ALONSO LUJAMBIO}

ción se institucionaliza en la citada Comisión. Se trata de un excelente estudio de caso que utiliza las herramientas del análisis institucional para entender un tema clave de la agenda de la investigación legislativa en Ciencia Política. El otro estudio, de Alvaro López Lara, investigador de la UAM, "Legislar y negociar: el quehacer de las Comisiones legislativas en México" (ponencia presentada en el Primer Congreso Nacional de Ciencia Política, Cd. de México, septiembre de 1996) analiza brevemente tres comisiones presididas por diputados de minoría en las LV y LVI Legislaturas (Asentamientos humanos, Justicia y Energéticos), para intentar comprender la importancia que en su momento tuvo el presidir comisiones desde la oposición minoritaria en el espacio del Poder Legislativo mexicano. ${ }^{7}$

El tema de la producción legislativa es de la mayor relevancia. En este campo sólo existen dos estudios terminados y otros dos en proceso. Entre los primeros está el de Jeffrey Weldon, "The Mexican Congress and the Presidency, 1917-1940" (ponencia presentada en la reunión anual de la American Political Science Association, en San Francisco, California, agosto de 1996). Gracias a la introducción del Diario de los Debates de la Cámara de Diputados desde 1917 a un CD-Rom, Jeffrey Weldon ha podido evidenciar con datos duros para el período 19171940 (iniciativas del presidente, iniciativas presentadas por el propio Congreso, enmiendas del Congreso a las iniciativas del presidente, vetos presidenciales a iniciativas aprobadas por el Congreso, superaciones congresionales de vetos presidenciales, etc.) la progresiva destrucción del poder del Congreso y el surgimiento del hiperpresidencialismo postrevolucionario. ${ }^{8} \mathrm{El}$ otro estudio concentra su atención en la producción legislativa de la era de los "diputados de partido" y es de

${ }^{7}$ Sobre el sistema de comisiones en el Senado, está prácticamente concluido el estudio de Gabriela Quijano, La evolución del sistema de comisiones en el Senado mexicano, 1934-1997 (ITAM, Departamento de Ciencias Sociales, tesis de Licenciatura en preparación).

${ }^{8}$ Sobre el particular puede consultarse también la obra de María Amparo Casar, Mexico: from Hyperpresidentialism to Presidentialism (Cambridge University, tesis de Doctorado en Ciencia Política, 1997). 
CIENCIA POLÍTICA Y PODER

Luisa Béjar, El papel de la Legislatura en el Sistema político mexicano, 1964-1979 (UNAM, tesis de Doctorado en Ciencia Política, 1995). Béjar nos ofrece evidencia, entre otras cosas, de que alrededor del $93 \%$ de las piezas de legislación aprobadas por el Congreso en el período estudiado tuvieron su origen en la oficina del Ejecutivo.

Algo sabemos, por otro lado, de la actividad legislativa de la izquierda independiente entendida como PCM-PSUM-PMS-PRD, gracias al ensayo de Adrián Michel y Dolores Prieto, "La izquierda independiente en la Cámara de Diputados, 1979-1994” (ITAM, mimeo, 1996). E1 estudio demuestra, entre otras cosas, que de las 130 iniciativas de ley que estas fuerzas introdujeron a la Cámara en el período estudiado, sólo el 15\% fueron dictaminadas en comisiones. De ellas, las aprobadas por el Pleno no sobrepasa el 5\%. Dato importante para el futuro: el PRI y la izquierda independiente sencillamente no han cooperado en la política legislativa de la etapa posterior a la Reforma Política de 1977. Sobre el PAN tenemos pocos datos y un estudio en proceso, el de Paola Martorelli, El trabajo legislativo del Partido Acción Nacional, 19461994 (ITAM, Departamento de Ciencias Sociales, tesis de Licenciatura en preparación), que muestra una especie de ciclicidad en la producción legislativa de Acción Nacional. La primera Legislatura en la que participa el PAN (la XI, 1946-1949) muestra una frenética actividad, que cae abruptamente en los cincuenta para repuntar notoriamente en la primera Legislatura de los "diputados de partido" (la XLVI, 19641967), para nuevamente inhibir su producción legislativa en la segunda década de los sesenta y a todo lo largo de los setenta. Los ochenta y los noventa son décadas, nuevamente, de alta productividad parlamentaria de Acción Nacional, si bien las tasas de rechazo de sus iniciativas no son relativamente bajas sino hasta finales de los ochenta y principios de los noventa.

Sin duda, la producción legislativa del Congreso de México va a alterarse ahora que vivimos bajo un gobierno dividido, esto es, donde el partido del titular del Poder Ejecutivo no cuenta con la mayoría absoluta de los escaños en una o ambas Cámaras del Congreso. Finalmente, esta situación, tan común en América Latina, se ha presentado 


\section{ALONSO LUJAMBIO}

en México en la LVII Legislatura (1997-2000). En torno a este punto de la agenda, el autor de estas líneas ha escrito un ensayo y ha editado una obra colectiva. En el primero, se discute qué tipo de estrategias pueden seguir los partidos políticos mexicanos para evitar parálisis institucional e ingobernabilidad en la relación entre poderes ("Presidentes y Congresos: los Estados Unidos, la experiencia latinoamericana y el Futuro Mexicano", en Federalismo y Congreso..., op. cit.). Por otro lado, apareció a mediados de 1997 un estudio comparativo de cinco estudios de caso (Baja California, Baja California Sur, Aguascalientes, Guanajuato y Chihuahua) en donde se analizan los Gobiernos divididos en la Federación mexicana (México: IFE-UAM-Colegio Nacional de Ciencias Políticas y Administración Pública, 1997) 9 desde el año de 1989, en que se presenta el primero. El libro analiza en cada caso las modificaciones que el gobierno dividido ha traido al sistema de comisiones, al Reglamento de sesiones, a la Ley Orgánica de los Congresos locales, así como los distintos tipos de coaliciones parlamentarias que los partidos han construido a partir de las distintas configuraciones de los sistemas de partidos locales, así como las producciones legislativas respectivas. Cabe señalar en este casi totalmente inexplorado rubro, relacionado con la comparación politológica de Congresos estatales en México, que existe una investigación que ha estudiado la evolución del tamaño de las legislaturas, entre otras las de los estados de nuestra Federación, para arribar a la conclusión de que dichas asambleas son pequeñas casi bajo cualquier parámetro con las que se les quiera medir, así como las consecuencias de subdimensionar

${ }^{9}$ Colaboran Tonatiuh Guillén, Marina Garmendia, Andrés Reyes, Alberto Aziz Nassif y Luis Miguel Rionda. Desde que se planeó el libro a la fecha, siete nuevos estados de la República se han sumado a la lista de Congresos Locales que viven bajo gobiernos divididos: Estado de México, Coahuila, Colima, Querétaro, Morelos, Sonora y Jalisco. Una importante obra, todavía en prensa, que analiza las relaciones Ejecutivo-Legislativo desde el arranque de la República Restaurada hasta la fundación del PNR en 1929, es la de Ignacio Marván Laborde, compilador, Episodios republicanos. Experiencia de Gobierno dividido en Mexico a partir de 1867, en prensa. 
CIENCIA POLIITICA Y PODER

a los cuerpos de deliberación legislativa (Rafael Vergara, El tamaño de las asambleas nacionales, ITAM, Departamento de Ciencias Sociales, tesis de Licenciatura en Ciencia Política, 1995).

Para terminar, se encuentra recientemente publicado un extenso estudio de orden lingüístico-sociológico, de Teresa Carbó, que analiza $E l$ discurso parlamentario mexicano entre 1920 y 1950 , especialmente el contenido de tres debates sobre el mundo indígena mexicano: el que se dio en 1920 sobre el Departamento de Educación y Cultura para la Raza Indígena, el de 1935 sobre el Departamento de Asuntos Indígenas, y el de 1948 sobre el Instituto Nacional Indigenista (México: Ciesas-El Colegio de México, 1996). ${ }^{10}$

\section{Conclusión}

En el pasado reciente, distintos proyectos que han intentado darle cierta continuidad a los estudios legislativos han fracasado. De la Revista Mexicana de Estudios Parlamentarios de la Cámara de Diputados, solo vieron la luz tres números a principios de los noventa. Por su parte, la revista Estudios Parlamentarios de la fundación Cambio XXI del PRI, sólo dio a conocer dos números, también a principios de esta década. La situación empieza, sin embargo, a cambiar. Un seminario permanente en el ITAM sobre estudios legislativos, diplomados en la UNAM y en la UAM-Iztapalapa, las revistas Quórum de la Cámara de Diputados y Estudios Parlamentarios del Congreso del Senado de la República, así como el ambicioso proyecto de investigación patrocinado por el Congreso y El Colegio de México, coordinado por los investigadores Romana Falcón, Juan Molinar Horcasitas y Jeffrey Weldon, ya revierten la precaria situación anterior. Estamos, pese a

${ }^{10}$ Cabe subrayar la existencia de otro libro, de reciente factura, que pese a no ser propiamente especializado, sí ofrece datos interesantes y compila información valiosa que se encontraba dispersa: Francisco Gil Villegas, coord., El Congreso mexicano. Estructura, organización, funcionamiento, análisis político (México: LV Legislatura de la Cámara de Diputad́os, 1994). 


\section{ALONSO LUJAMBIO}

todo, y todavía, entre el pasado y el futuro. Sin duda, la dinámica política mexicana, y la traducción de ésta en poderes legislativos crecientemente plurales tanto a nivel federal como estatal, producen un caldo de cultivo propicio para que la ciencia política mexicana se involucre más en los estudios parlamentarios. La situación es de cualquier modo promisoria y existen plenas razones para justificar el optimismo. 